\title{
Genomics-based dissection of the cross-talk of chloroplasts with the nucleus and mitochondria in Arabidopsis
}

\author{
Dario Leister* \\ Abteilung für Pflanzenzüchtung und Genetik; Max-Planck-Institut für Züchtungsforschung, Carl-von-Linné Weg 10, D-50829 Köln, Germany
}

\author{
Received 12 October 2004; accepted 25 March 2005 \\ Available online 23 May 2005 \\ Received by K. Wolf
}

\begin{abstract}
Post-endosymbiotic evolution of chloroplasts was characterized by a massive transfer of cyanobacterial genes to the nucleus, followed by re-routing of many of their encoded proteins. In consequence, most plastid proteins are nucleus-encoded, enabling an anterograde (nucleusto-plastid) control of the organelle. The regulation of chloroplast functions includes also cross-talk between chloroplasts and mitochondria, as well as retrograde (plastid-to-nucleus) signalling. Genetic analyses reveal that redox state, flux through the chlorophyll biosynthetic pathway, sugar sensing and reactive oxygen species contribute to retrograde signalling. However, the identity of the messenger molecule(s) remains largely unknown. Novel facets of the chloroplast-mitochondrion cross-talk have been revealed by the characterization of mitochondrial mutants affected in chloroplast properties. Studies of the nuclear chloroplast transcriptome imply the existence of at least three distinct types of transcriptional regulation: a master switch, acting in a binary mode by either inducing or repressing the same large set of genes; a "mixed response" with about equal numbers of up- and down-regulated genes; and mechanisms supporting the specific co-regulation of nuclear genes for photosynthesis and for plastid gene expression. The recent discovery of the latter mode of control highlights a possibly ancient route to co-ordinate chloroplast and nuclear genome expression.
\end{abstract}

(C) 2005 Elsevier B.V. All rights reserved.

Keywords: Anterograde signalling; Mutant; Photosynthesis; Plastid signal; Retrograde signalling; Transcriptome

\section{Introduction}

In plants, genetic information is distributed between three compartments. Chloroplasts and mitochondria are of endosymbiotic origin, but contain only relatively few proteincoding genes - between 3 (Plasmodium mitochondrion) and 209 (Porphyra chloroplast). Since their contemporary prokaryotic relatives possess several thousands genes, this reflects an ancient, extensive transfer of their original

Abbreviations: Cue, chlorophyll $a / b$ binding protein under-expression; Gun, genomes uncoupled; LHC, light-harvesting complex; PSI (II), photosystem I (II); ROS, reactive oxygen species; Tic, translocon at the inner envelope of the chloroplast; Tim, translocon at the inner envelope of the mitochondrion; Toc, translocon at the outer envelope of the chloroplast; Tom, translocon at the outer envelope of the mitochondrion.

* Tel.: +49 221 5062415; fax: +49 2215062413 .

E-mail address: 1eister@mpiz-koeln.mpg.de.

0378-1119/\$ - see front matter (C) 2005 Elsevier B.V. All rights reserved. doi:10.1016/j.gene.2005.03.039 genetic material to the nucleus. In consequence, the majority of mitochondrial and chloroplast proteins are nucleusencoded, synthesized in the cytoplasm, and post-translationally imported into the organelles. The location of the genes encoding organelle proteins in different compartments implies the existence of mechanisms that serve to integrate nuclear and organelle gene expression, including interorganellar signalling and the co-ordinated expression of sets of nuclear genes coding for organelle proteins (in the following designated as "nuclear organelle genes"). The cross-talk between organelles includes both anterograde (nucleus-to-organelle) and retrograde (organelle-to-nucleus) controls. Anterograde mechanisms co-ordinate gene expression in chloroplasts and mitochondria, and are receptive to endogenous and environmental signals perceived by the nucleus. Retrograde signalling regulates the expression of nuclear organelle genes in response to the metabolic and developmental state of the organelle. Besides the cross-talk 
between chloroplasts/mitochondria and the nucleus, chloroplast-mitochondrion communication has been established during plant evolution to co-ordinate the activities of the two organelles which exhibit a high level of metabolic interdependence.

Here, I summarize the state and recent progress in the fields of (i) inter-organellar gene flow, (ii) anterograde mechanisms, (iii) transcriptional regulation of nuclear chloroplast genes, with emphasis on retrograde mechanisms, (iv) chloroplast-mitochondrion cross-talk, and (v) the transcription-based co-ordination of the expression of nuclear and plastid genes.

\section{Inter-organellar DNA transfer and protein re-routing}

Several cases of nucleus-to-mitochondrion and plastidto-mitochondrion DNA transfer have been noted in flowering plants. On the contrary, migration of chondrome-derived sequences to plastid DNA has not been described yet. Between $1 \%$ and $6 \%$ of the mitochondrial genomes of Arabidopsis thaliana, sugar beet and rice derive from plastid DNA, while retrotransposons seem to be the source of nucleus-derived mitochondrial DNA (Unseld et al., 1997; Kubo et al., 2000; Notsu et al., 2002). However, only very few of such promiscuous DNA sequences are functional, namely plastid-derived mitochondrial tRNA genes.

Organelle-to-nucleus DNA transfer is a fundamental, ongoing evolutionary process which paved the way for organelle-nucleus cross-talk. Ample evidence exists that DNA is directly transferred, at high frequency, from organelle genomes to the nucleus (Richly and Leister, 2004a,b) and that even entire organelle chromosomes have been transferred (Timmis et al., 2004). Present-day organelle-to-nucleus DNA transfer contributes to genetic variation via a mutation-inducing mechanism (Richly and Leister, 2004a; Timmis et al., 2004). During the early phase of organelle evolution, the same mechanism has resulted in a massive re-location of organelle genes to the nucleus: it has been estimated that of the $\sim 4500$ nuclear $A$. thaliana genes of cyanobacterial descent, only $\sim 1300$ code for chloroplast-targeted proteins (Martin et al., 2002). This implies that the successful establishment of the chloroplast organelle was paralleled by a substantial change in the composition of the proteomes of all cellular compartments (Leister and Schneider, 2004). At present, the Arabidopsis chloroplast is predicted to contain between 2500 and 3000 proteins, of which about half is of cyanobacterial origin (Richly and Leister, 2004c).

\section{Anterograde mechanisms}

The large majority of chloroplast and mitochondrial proteins are nucleus-encoded. Multiple levels of anterograde control of organelle properties, including organelle gene expression, exist. (i) Modulation of the level of transcripts of nuclear chloroplast genes. This mechanism is suitable to control the abundance of many chloroplast proteins (Kleffmann et al., 2004). (ii) Post-translational import of proteins into chloroplasts and mitochondria facilitated by the Tic/Toc and Tim/Tom complexes, respectively. Isoforms of these translocons exist with different substrate specificity, possibly allowing the accumulation of tissue-specific plastid and mitochondrial proteomes (Lister et al., 2004; Soll and Schleiff, 2004). Regulation of protein import into chloroplasts, moreover, seems to involve sensing and reacting to the redox state of the organelle (Küchler et al., 2002). (iii) Transcription, transcript editing, maturation and processing, as well as the translation of organelle-encoded proteins and their auto-regulation. This is, in large parts, mediated by nuclear-encoded factors, allowing nuclear control of the expression of organelle genes (Leon et al., 1998; Choquet and Wollman, 2002). (iv) Post-translational events, such as the assembly of the multi-protein complexes of the thylakoid membrane or of the inner envelope of mitochondria. These processes require nuclear-encoded assembly factors (Grivell et al., 1999; Vothknecht and Westhoff, 2001). (v) Organelle development, for instance organelle division, which is tightly controlled by nuclear-encoded proteins (Osteryoung and Nunnari, 2003).

Current data support the conclusion that the regulation of plastome and chondrome gene expression occurs mainly at the post-transcriptional level (Leon et al., 1998; Rochaix, 2001).

\section{Mutants and retrograde signalling}

\subsection{Redox signalling}

Early evidence that nuclear genes are regulated by plastid-derived signals came from studies of photo-oxidized plants, which showed decreased expression of nuclear photosynthetic genes. Regulation occurs frequently at the transcriptional level, and the Lhcb genes, coding for lightharvesting proteins of photosystem II, were found to be the most down-regulated. The transcriptional response of selected nuclear photosynthetic genes to variation in the excitation state of the two photosystems showed that the redox state of the plastoquinone pool controls the activity of the plastocyanin promoter (Pfannschmidt et al., 2001). The Arabidopsis "chlorophyll $a / b$ binding protein under-expression" mutant cuel offered additional evidence for the involvement of the redox state of the plastoquinone pool in the regulation of nuclear photosynthetic genes (Streatfield et al., 1999): lack of the CUE1-encoded phosphoenolpyruvate/phosphate translocator (PPT1) in the inner chloroplast envelope results in a reduced flux through the shikimate pathway and in a decrease in the plastoqinone pool, associated with an altered redox state of the thylakoids. The redox state of the stromal electron acceptors of PSI, in 
fact, seems to be crucial for the regulation of $L h c b$ genes (Pursiheimo et al., 2001).

\subsection{Tetrapyrrole signalling}

In addition to redox signalling, the chlorophyll biosynthetic pathway has been associated with the control of nuclear gene expression. In Chlamydomonas, intermediates in the chlorophyll biosynthetic pathway modulate the accumulation of transcripts of several nuclear chloroplast genes (Johanningmeier and Howell, 1984; Kropat et al., 1997). Arabidopsis mutants that do not react to norflurazoninduced photo-oxidative damage by repression of $L h c b$ transcription (genomes uncoupled 1-5: gun 1-5) were affected in genes encoding proteins involved in tetrapyrrole metabolism: the products of GUN2/HY1 and GUN3/HY2 contribute to heme degradation in the "Fe branch" of tetrapyrrole biosynthesis (Mochizuki et al., 2001), GUN5 encodes the CHL H subunit of the Mg-chelatase (Mochizuki et al., 2001), and GUN4 binds product and substrate of Mgchelatase, and activates Mg-chelatase (Larkin et al., 2003).

Experimental evidence for a strict correlation between enzyme activities in the "Mg branch" of tetrapyrrole biosynthesis and gene expression of certain nuclear genes for photosynthesis or tetrapyrrole formation (Papenbrock et al., 2000; Yaronskaya et al., 2003; Wilde et al., 2004; Alawady and Grimm, 2004) substantiated the gun mutant analysis, prompting proposals in favour of a role of $\mathrm{Mg}$ porphyrins and/or enzymes of this biosynthetic pathway as sensors, or as mediating a plastid signal. GUN5 was originally suggested to act as a sensor (Mochizuki et al., 2001), but the idea was recently revised in favour of the tetrapyrrole intermediate Mg-protoporphyrin IX, a proposed signalling molecule between chloroplast and nucleus (Strand et al., 2003; Fig. 1). In this model, GUN4 is thought to participate in signalling by regulating $\mathrm{Mg}$ protoporphyrin IX synthesis or trafficking (Larkin et al., 2003; Wilde et al., 2004), while an additional chloroplast protein, LAF6/ABC1, which serves as a component of the phytochrome A signalling pathway, is thought to be involved in the transport and correct distribution of protoporphyrin IX (Moller et al., 2001).

\subsection{Signalling involving sugars or reactive oxygen species}

Two additional classes of molecules have been associated with retrograde signalling: sugars and reactive oxygen species (Fig. 1). The regulatory effect of sugars on the

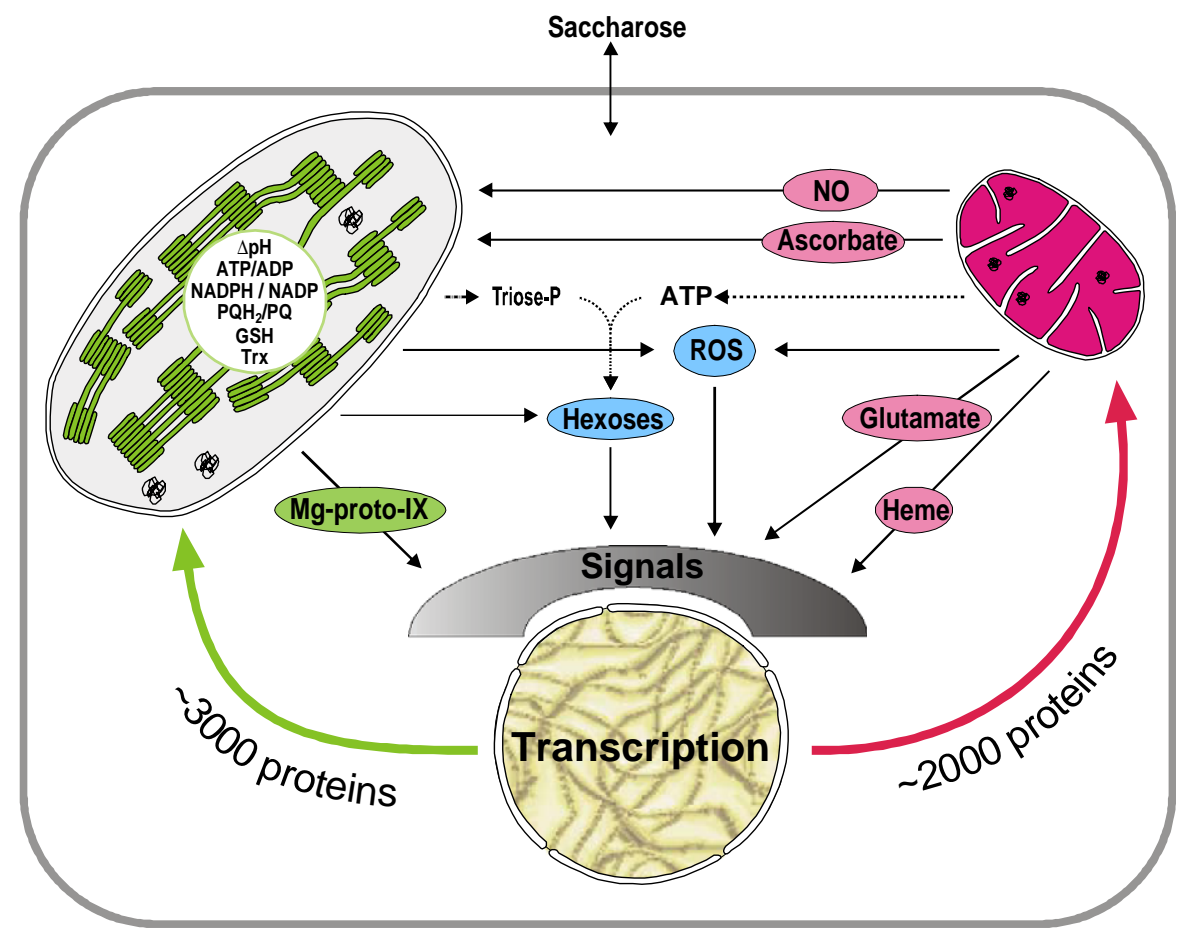

Fig. 1. Overview of cross-talk between different compartments in higher plants. Green ovals indicate candidate plastid-derived signals, pink symbolize mitochondrion-derived molecules, and blue ones (hexoses and reactive oxygen species (ROS)) highlight signals from both organelles. Glutamate and heme were shown to function as mitochondrial signals in yeast (Forsburg and Guarente, 1989; Butow, 2002); in plants, heme is synthesized also in plastids. Mitochondrial 2-oxoglutarate has been also associated with biochemical and retrograde signalling in plants (Hodges, 2002) (not shown). Nitric oxide and ascorbate have been proposed to act as mitochondrion-to-plastid signals (reviewed in: Raghavendra and Padmasree, 2003). Numbers of chloroplast $(\sim 3000)$ and mitochondrial $(\sim 2000)$ proteins encoded in the nucleus are according to Richly and Leister (2004c). A selection of chloroplast properties, discussed in the frame of experiments addressing signalling within chloroplasts and with the potential to be also involved in retrograde signalling, is listed in the green circle: $\Delta \mathrm{pH}, \mathrm{pH}$-gradient between thylakoid lumen and stroma; ATP/ADP, ratio of ATP and ADP; NADPH/NADP, ratio of NADPH and NADP; PQH/PQ, redox state of the plastoquinone pool; GSH, glutathione; Trx, thioredoxins. 
expression of nuclear photosynthetic genes and on plant metabolism is well known (reviewed in: Rolland et al., 2002). In general, a low sugar status enhances photosynthesis and induces reserve mobilization and export, whereas an abundant supply of sugars promotes growth and carbohydrate storage. Dark-to-light shift experiments with the Arabidopsis flu mutant revealed that singlet oxygen, a nonradical reactive oxygen species, was confined to plastids and activates several stress-response pathways (op den Camp et al., 2003). The biological activity of this reactive oxygen species is highly specific, and seems to depend from the chemical identity of the molecule and/or the site at which it is generated (op den Camp et al., 2003). Also photorespiratory $\mathrm{H}_{2} \mathrm{O}_{2}$ has been suggested to have a direct impact on the transcription of nuclear genes (Vandenabeele et al., 2004).

\section{Cross-talk between chloroplast and mitochondrion}

The complex metabolic interdependence of chloroplasts and mitochondria is well documented (Raghavendra and Padmasree, 2003). For instance, photosynthesis provides substrates for mitochondrial respiration, but depends itself on a range of compounds synthesized by mitochondria, including ATP in the dark. Moreover, mitochondrial respiration protects photosynthesis against photoinhibition by dissipating redox equivalents exported from the chloroplasts.

Genetic studies have significantly contributed to the dissection of chloroplast-mitochondrion cross-talk. The existence of suppressor genes in the chloroplast that act on a mitochondrial mutation in $C$. reinhardtii suggests exchange of tRNAs between the two organelles (Bennoun and Delosme, 1999). In the barley albostrians mutant, the absence of chloroplast activity in an otherwise fully differentiated leaf tissue leads to an increase in mitochondrial gene copy number and an elevated level of mitochondrial transcripts (Hedtke et al., 1999). Vice versa, mutations affecting specific mitochondrial proteins have been analyzed for their effects on chloroplast properties, like $g d c$ of potato (Heineke et al., 2001) and barley (Igamberdiev et al., 2001). Both mutants exhibit a lowered glycine decarboxylase activity and are impaired in photorespiration, leading to an over-reduction and over-energization of the chloroplast. In the tobacco CMSII mutant, which lacks the major mitochondrial NADH dehydrogenase (Complex I), the rate of photosynthesis is decreased, notably during dark-light transitions or when carbon fixation and photorespiration are simultaneously active (Sabar et al., 2000; Dutilleul et al., 2003a). In the same mutant, modulation of nuclear gene expression maintains whole cell redox balance (Dutilleul et al., 2003b). The analysis of the prors 1 mutant of Arabidopsis, defective in an organelle-targeted tRNA synthetase, shows that nuclear photosynthetic genes are specifically down-regulated (P. Pesaresi and D. Leister, unpublished results), indicating that mitochondrion-chloroplast crosstalk might involve re-programming of the expression of nuclear chloroplast genes.

\section{The global picture: transcription pattern of the nuclear chloroplast genome}

Complementary to the genetic approach outlined in Section 4, the systematic analysis of mRNA expression profiles of nuclear organelle genes allows to propose mechanisms active at the "receiver" side of retrograde signalling. The most advanced and detailed study of the expression of nuclear organelle genes, so far, analyzed the transcript accumulation of more than 3000 nuclear genes of Arabidopsis, most of them coding for chloroplast proteins (Richly et al., 2003; Biehl et al., 2004), studied under 101 different genetic or environmental conditions. This approach proved efficient in revealing compensatory mechanisms acting in response to altered states of the chloroplast (reviewed in: Leister and Schneider, 2003), and its full potential was appreciated when the 101 gene expression profiles were compared. Different layers of transcriptional control of nuclear chloroplast genes became evident. (i) A "master switch", acting in a binary mode by either inducing or repressing the same large set of genes (Fig. 2A), and which is triggered by at least half of the 101 conditions investigated (Richly et al., 2003; Biehl et al., 2004). (ii) A "mixed response", induced by the other half of conditions, with about equal numbers of up- and down-regulated genes (Richly et al., 2003). This response can be further divided into three distinct sub-groups (Biehl et al., 2004; Fig. 2A). (iii) Two regulons (regulons 1 and 2, see Fig. 2A), containing 207 genes that mostly code for proteins of the photosystems or for plastome gene expression, escape the transcriptional master switch, thus indicating the existence of an additional, distinct type of transcriptional regulation.

The transcriptional behaviour of regulons 1 and 2 implies the existence of a mechanism that co-ordinates the expression of plastome- and nucleus-encoded proteins of the photosystems (Biehl et al., 2004). In such complexes, the increase of nuclear-encoded photosystem proteins has to be matched by a similar modification of the level of plastome-encoded proteins. This adjustment should be achieved by a nucleus-dependent increase in plastid ribosomal proteins-ultimately of the number of ribosomes-in support of a superior translational capacity of the chloroplast. Interestingly, of the 207 genes associated with this master-switch-independent mode of transcriptional regulation, a substantial fraction can be traced back to the cyanobacterial endosymbiont (Fig. 2B). This might indicate that this type of transcriptional regulation has been established relatively early after endosymbiosis, when almost all proteins of the proto-chloroplast were of cyanobacterial origin. It cannot be excluded, however, that this large fraction of cyanobacterial genes could be due to 
A 101 conditions

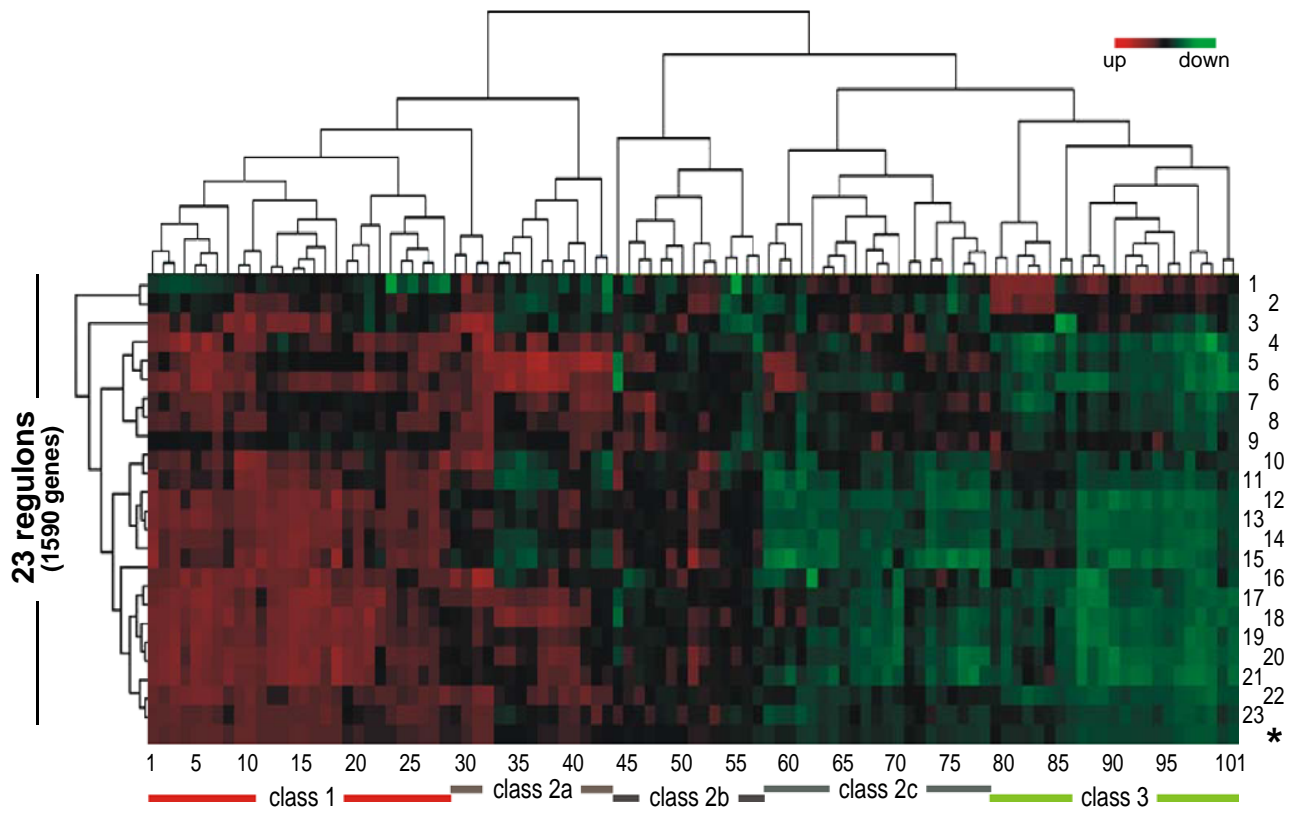

B number of genes

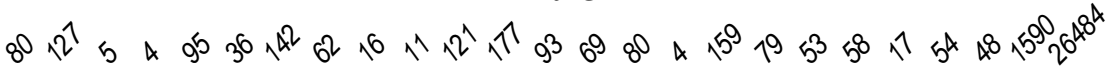

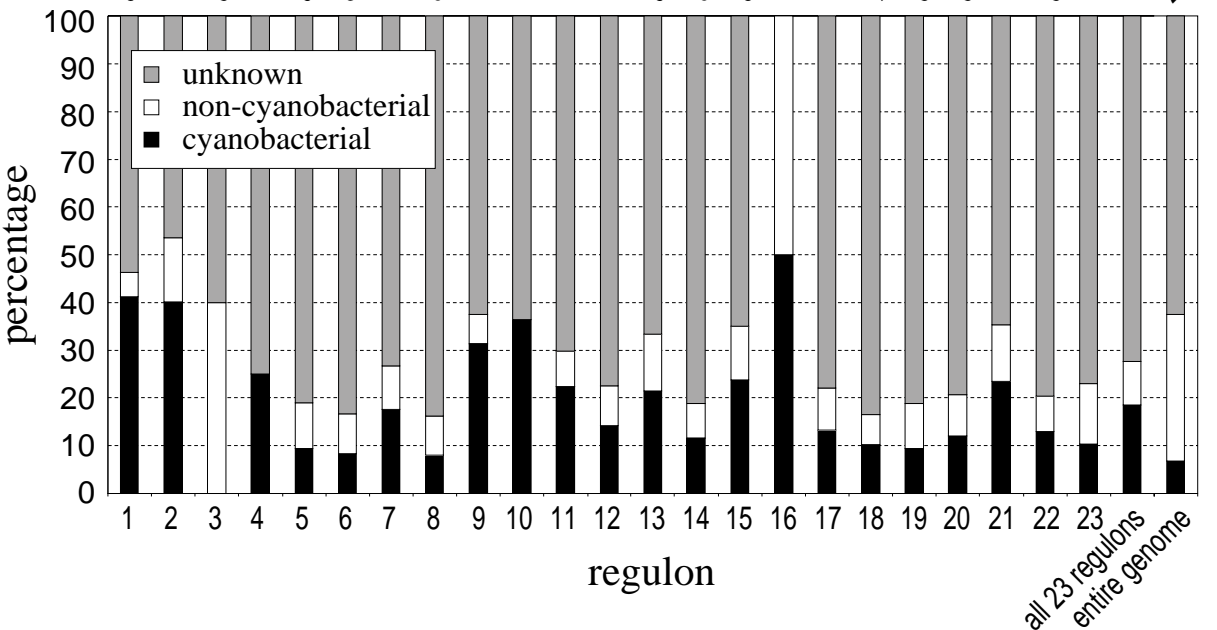

Fig. 2. Analysis of the nuclear chloroplast transcriptome. (A) Clustering of the expression profiles of 1590 genes, most of them coding for chloroplast proteins, from plants grown under 101 different conditions, resulted in the identification of 23 groups of co-expressed genes (regulons) (Biehl et al., 2004). Colours indicate up-(red) or down-(green) regulation, whereas grey lines indicate missing values. The cladograms on top and at left describe the relatedness of transcriptome responses in terms of conditions, or as reaction of individual genes, respectively. The numbers at the bottom of the cluster refer to the 101 conditions; designation of regulons is displayed at the right, and the last row (asterisk) indicates the average of all 1590 genes. Regulons 1 and 2 (see Section 6 ) are most distantly related to the average of all regulons and contain mostly genes involved in photosynthesis or chloroplast gene expression. Designation of response classes (class 1 and 3, "master switch"; class 2, "mixed response") is according to Richly et al. (2003) and Biehl et al. (2004) (see Section 6). (B) Phylogenetic composition of the 23 regulons. Genes contained in the 23 regulons were classified as "cyanobacterial", "non-cyanobacterial" and "unknown" according to Martin et al. (2002). The actual fraction of cyanobacterial and non-cyanobacterial genes might be substantially larger because only for 9368 Arabidopsis genes the phylogeny has been unambiguously determined by Martin et al. (2002). Note that of all regulons of relevant size, regulons 1 and 2 contain the largest fraction of cyanobacterial genes.

the conservation-in terms of mechanisms and proteins involved-of photosynthesis and plastid gene expression between chloroplasts and cyanobacteria.

In summary, at least three distinct responses of the nuclear chloroplast transcriptome exist: master switch (classes 1 and 3; Fig. 1A), mixed response (class 2), and the co-variation of transcripts of photosynthetic and plastid gene expression genes (regulons 1 and 2). Since the mixed response can be further divided, in total even five different mechanisms of response might operate. Can these distinct 
responses be correlated with any of the proposed retrograde signalling pathways: "redox", "sugar", "reactive oxygen species" and "tetrapyrrole"? The answer is positive: the gun mutations ("tetrapyrrole") induce class 1 response, whereas the cuel mutant ("redox") results in a class 3 response (Richly et al., 2003). High-light stress, supposed to cause "reactive oxygen species"-type signalling, sucrose feeding ("sugar signalling"), as well as many other genetic and environmental conditions, also result in class 1 response (Biehl et al., 2004). This finding makes it difficult to assign the distinct classes of transcriptional response to particular signalling pathways. It is conceivable that most treatments or mutations have secondary effects and trigger more than one retrograde signalling pathway. In this context, a careful design of DNA-array experiments is at need; for instance, the comparison of mRNA expression profiles of high-light treated control and catalase-deficient plants allowed to discriminate $\mathrm{H}_{2} \mathrm{O}_{2}$-dependent and -independent types of transcription response during light stress (Vandenabeele et al., 2004).

\section{Conclusions}

Evolutionary organelle-to-nucleus transfer of genes enabled re-location of proteins from their original compartments and the co-ordination of the expression of proteins of different compartments mediated by nuclear transcription (see below). In this situation, most of the organelle proteins are not encoded and synthesized in the compartment where they have to function. Because of this, nuclear organelle genes were required to adjust their expression in dependence of the organelle state, and the retrograde control of nuclear gene expression emerged. Our understanding of retrograde signalling, however, is still far from being complete. A number of chloroplast signals have been put forward, most of them connected to the organelle's redox state or the chlorophyll biosynthetic pathway. Reactive oxygen species, a harmful by-product of photosynthesis and of mitochondrial respiration, emerge as candidates for retrograde signalling molecules. Since reactive oxygen species are generated by both organelles, they might allow co-ordination at the nuclear transcriptional level of plastid and mitochondrial performances. Most regulons identified by Biehl et al. (2004) contain both nuclear chloroplast and nuclear mitochondrial genes, allowing co-regulation of chloroplast and mitochondrial properties. Currently, we have no knowledge of the "receiver side" of retrograde signalling, i.e. of the identity of the nuclear proteins mediating organelle-state-induced transcription changes. Although specific promoter elements have been identified (Strand et al., 2003), genetic screens were, so far, unsuccessful in identifying any nuclear protein involved in retrograde signalling. This suggests either a high level of redundancy, or the essentiality of the corresponding factors. The identification of downstream factors in retrograde signalling is likely to require novel genetic screens in Arabidopsis and/or the consideration of other plant species. The recent analysis of the nuclear chloroplast transcriptome clearly indicates that different layers of nuclear transcriptional control-corresponding to several distinct types of plastid signals-should exist, adding to the complexity of intra-cellular signalling in flowering plants.

\section{Acknowledgements}

Francesco Salamini, Paolo Pesaresi, Bernhard Grimm and Maarten Koornneef are gratefully acknowledged for critical reading of the manuscript.

\section{References}

Alawady, A., Grimm, B., 2004. Tobacco Mg protoporphyrin IX methyltransferase is involved in inverse activation of $\mathrm{Mg}$ porphyrin and protoheme synthesis. Plant J. 41, 282-290.

Bennoun, P., Delosme, M., 1999. Chloroplast suppressors that act on a mitochondrial mutation in Chlamydomonas reinhardtii. Mol. Gen. Genet. 262, 85-89.

Biehl, A., Richly, E., Noutsos, C., Salamini, F., Leister, D., 2004. Analysis of 101 nuclear transcriptomes reveals 23 distinct regulons and their relationship to metabolism, chromosomal gene distribution and coordination of nuclear and plastid gene expression. Gene 344, 33-41.

Butow, R.A., 2002. Cellular responses to mitochondrial dysfunction: it's not always downhill. Cell Death Differ. 9, 1043-1045.

Choquet, Y., Wollman, F.A., 2002. Translational regulations as specific traits of chloroplast gene expression. FEBS Lett. 529, 39-42.

Dutilleul, C., Driscoll, S., Cornic, G., De Paepe, R., Foyer, C.H., Noctor, G., 2003a. Functional mitochondrial complex I is required by tobacco leaves for optimal photosynthetic performance in photorespiratory conditions and during transients. Plant Physiol. 131, 264-275.

Dutilleul, C., et al., 2003b. Leaf mitochondria modulate whole cell redox homeostasis, set antioxidant capacity, and determine stress resistance through altered signaling and diurnal regulation. Plant Cell $15,1212-1226$.

Forsburg, S.L., Guarente, L., 1989. Communication between mitochondria and the nucleus in regulation of cytochrome genes in the yeast Saccharomyces cerevisiae. Annu. Rev. Cell Biol. 5, 153-180.

Grivell, L.A., et al., 1999. Mitochondrial assembly in yeast. FEBS Lett. 452, 57-60.

Hedtke, B., Wagner, I., Borner, T., Hess, W.R., 1999. Inter-organellar crosstalk in higher plants: impaired chloroplast development affects mitochondrial gene and transcript levels. Plant J. 19, 635-643.

Heineke, D., Bykova, N., Gardestrom, P., Bauwe, H., 2001. Metabolic response of potato plants to an antisense reduction of the P-protein of glycine decarboxylase. Planta 212, 880-887.

Hodges, M., 2002. Enzyme redundancy and the importance of 2-oxoglutarate in plant ammonium assimilation. J. Exp. Bot. 53, 905-916.

Igamberdiev, A.U., Bykova, N.V., Lea, P.J., Gardestrom, P., 2001. The role of photorespiration in redox and energy balance of photosynthetic plant cells: a study with a barley mutant deficient in glycine decarboxylase. Physiol. Plant. 111, 427-438.

Johanningmeier, U., Howell, S.H., 1984. Regulation of light-harvesting chlorophyll-binding protein mRNA accumulation in Chlamydomonas reinhardtii. Possible involvement of chlorophyll synthesis precursors. J. Biol. Chem. 259, 13541-13549.

Kleffmann, T., et al., 2004. The Arabidopsis thaliana chloroplast proteome reveals pathway abundance and novel protein functions. Curr. Biol. 14, $354-362$ 
Kropat, J., Oster, U., Rüdiger, W., Beck, C.F., 1997. Chlorophyll precursors are signals of chloroplast origin involved in light induction of nuclear heat-shock genes. Proc. Natl. Acad. Sci. U. S. A. 94, $14168-14172$.

Kubo, T., Nishizawa, S., Sugawara, A., Itchoda, N., Estiati, A., Mikami, T., 2000. The complete nucleotide sequence of the mitochondrial genome of sugar beet, (Beta vulgaris L.) reveals a novel gene for tRNA $^{\text {Cys }}($ GCA). Nucleic Acids Res. 28, 2571-2576.

Küchler, M., Decker, S., Hormann, F., Soll, J., Heins, L., 2002. Protein import into chloroplasts involves redox-regulated proteins. EMBO J. 21, 6136-6145.

Larkin, R.M., Alonso, J.M., Ecker, J.R., Chory, J., 2003. GUN4, a regulator of chlorophyll synthesis and intracellular signaling. Science 299, 902-906.

Leister, D., Schneider, A., 2003. From genes to photosynthesis in Arabidopsis thaliana. Int. Rev. Cytol. 228, 31-83.

Leister, D., Schneider, A., 2004. Evolutionary contribution of plastid genes to plant nuclear genomes and its effects on composition of the proteomes of all cellular compartments. In: Hirt, R.P., Horner, R.P., Horner, D.S. (Eds.), Organelle, Genomes and Eukaryote Phylogeny: An Evolutionary Synthesis in the Age of Genomics. The Systematics Association Special Volume Series, vol. 68. CRC Press, Boca Raton, pp. 237-255.

Leon, P., Arroyo, A., Mackenzie, S., 1998. Nuclear control of plastid and mitochondrial development in higher plants. Annu. Rev. Plant Physiol. Plant Mol. Biol. 49, 453-480.

Lister, R., et al., 2004. A transcriptomic and proteomic characterization of the Arabidopsis mitochondrial protein import apparatus and its response to mitochondrial dysfunction. Plant Physiol. 134, 777-789.

Martin, W., et al., 2002. Evolutionary analysis of Arabidopsis, cyanobacterial, and chloroplast genomes reveals plastid phylogeny and thousands of cyanobacterial genes in the nucleus. Proc. Natl. Acad. Sci. U. S. A. 99, 12246-12251.

Mochizuki, N., Brusslan, J.A., Larkin, R., Nagatani, A., Chory, J., 2001. Arabidopsis genomes uncoupled 5 (GUN5) mutant reveals the involvement of Mg-chelatase $\mathrm{H}$ subunit in plastid-to-nucleus signal transduction. Proc. Natl. Acad. Sci. U. S. A. 98, 2053-2058.

Moller, S.G., Kunkel, T., Chua, N.H., 2001. A plastidic ABC protein involved in intercompartmental communication of light signaling. Genes Dev. 15, 90-103.

Notsu, Y., et al., 2002. The complete sequence of the rice (Oryza sativa L.) mitochondrial genome: frequent DNA sequence acquisition and loss during the evolution of flowering plants. Mol. Genet. Genomics 268, $434-445$.

op den Camp, R.G., et al., 2003. Rapid induction of distinct stress responses after the release of singlet oxygen in Arabidopsis. Plant Cell 15, 2320-2332.

Osteryoung, K.W., Nunnari, J., 2003. The division of endosymbiotic organelles. Science 302, 1698-1704.

Papenbrock, J., Mock, H.P., Tanaka, R., Kruse, E., Grimm, B., 2000. Role of magnesium chelatase activity in the early steps of the tetrapyrrole biosynthetic pathway. Plant Physiol. 122, 1161-1169.

Pfannschmidt, T., Schutze, K., Brost, M., Oelmuller, R., 2001. A novel mechanism of nuclear photosynthesis gene regulation by redox signals from the chloroplast during photosystem stoichiometry adjustment J. Biol. Chem. 276, 36125-36130.

Pursiheimo, S., Mulo, P., Rintamäki, E., Aro, E.M., 2001. Coregulation of light-harvesting complex II phosphorylation and lhcb mRNA accumulation in winter rye. Plant J. 26, 317-327.

Raghavendra, A.S., Padmasree, K., 2003. Beneficial interactions of mitochondrial metabolism with photosynthetic carbon assimilation. Trends Plant Sci. 8, 546-553.

Richly, E., Leister, D., 2004a. NUPTs in sequenced eukaryotes and their genomic organization in relation to NUMTs. Mol. Biol. Evol. 21, $1972-1980$

Richly, E., Leister, D., 2004b. NUMTs in sequenced eukaryotic genomes. Mol. Biol. Evol. 21, 1081-1084.

Richly, E., Leister, D., 2004c. An improved prediction of chloroplast proteins reveals diversities and commonalities in the chloroplast proteomes of Arabidopsis and rice. Gene 329, 11-16.

Richly, E., et al., 2003. Covariations in the nuclear chloroplast transcriptome reveal a regulatory master-switch. EMBO Rep. 4, 491-498.

Rochaix, J.D., 2001. Posttranscriptional control of chloroplast gene expression. From RNA to photosynthetic complex. Plant Physiol. 125, $142-144$.

Rolland, F., Moore, B., Sheen, J., 2002. Sugar sensing and signaling in plants. Plant Cell 14, S185-S205 (Suppl.).

Sabar, M., De Paepe, R., de Kouchkovsky, Y., 2000. Complex I impairment, respiratory compensations, and photosynthetic decrease in nuclear and mitochondrial male sterile mutants of Nicotiana sylvestris. Plant Physiol. 124, 1239-1250.

Soll, J., Schleiff, E., 2004. Protein import into chloroplasts. Nat. Rev., Mol Cell Biol. 5, 198-208.

Strand, A., Asami, T., Alonso, J., Ecker, J.R., Chory, J., 2003. Chloroplast to nucleus communication triggered by accumulation of Mg-protoporphyrin IX. Nature 421, 79-83.

Streatfield, S.J., et al., 1999. The phosphoenolpyruvate/phosphate translocator is required for phenolic metabolism, palisade cell development, and plastid-dependent nuclear gene expression. Plant Cell 11, $1609-1622$

Timmis, J.N., Ayliffe, M.A., Huang, C.Y., Martin, W., 2004. Endosymbiotic gene transfer: organelle genomes forge eukaryotic chromosomes. Nat. Rev., Genet. 5, 123-135.

Unseld, M., Marienfeld, J.R., Brandt, P., Brennicke, A., 1997. The mitochondrial genome of Arabidopsis thaliana contains 57 genes in 366,924 nucleotides. Nat. Genet. 15, 57-61.

Vandenabeele, S., et al., 2004. Catalase deficiency drastically affects gene expression induced by high light in Arabidopsis thaliana. Plant J. 39, $45-58$.

Vothknecht, U.C., Westhoff, P., 2001. Biogenesis and origin of thylakoid membranes. Biochim. Biophys. Acta 1541, 91-101.

Wilde, A., Mikolajczyk, S., Alawady, A., Lokstein, H., Grimm, B., 2004. The gun4 gene is essential for cyanobacterial porphyrin metabolism. FEBS Lett. 571, 119-123.

Yaronskaya, E., Ziemann, V., Walter, G., Averina, N., Börner, T., Grimm, B., 2003. Metabolic control of the tetrapyrrole biosynthetic pathway for porphyrin distribution in the barley mutant albostrians. Plant J. 35, 512-522. 